\title{
Encircled Angular Flux Representation of the Modal Power Distribution and Its Behavior in a Step Index Multimode Fiber
}

\author{
Manabu Kagami, Member, IEEE, Member, OSA, Akari Kawasaki, Masatoshi Yonemura, Makoto Nakai, \\ Pablo V. Mena, Member, IEEE, and David R. Selviah, Member, IEEE
}

\begin{abstract}
The optical properties of inline optical components inserted at the input to a multimode optical fiber (MMF) strongly affect the propagating modal power distribution (MPD). To realize stable systems with predictable reproducible performance and to encourage widespread use of MMF systems, improvements to the system design process and to the characterization process need to be made and new interface standards need to be defined. To this end, we have developed a new reproducible MPD measurement and representation together with its theoretical definition. By modifying the encircled flux (EF) representation, which is based on the near-field pattern of a graded-index multimode optical fiber (GIMMF), we define the encircled angular flux (EAF) for step-index multimode optical fibers (SI-MMFs) based on their far-field patterns (FFPs). Using a SI-MMF, which is used for low cost short distance interconnects, as an example, we show the changes in the MPD along the fiber, reveal an unusual insertion loss-increasing phenomenon due to the evanescent tails of the core modes extending into the cladding, and characterize the equilibrium mode distribution (EMD) in the fiber. The EAF representation enables these phenomena to be quantified. We also propose an EAF template that consists of the target EMD and its tolerance. If device system designers use the EAF template to set the launch conditions, they can perform a fair assessment of the components, and they can design the system performance even if some components are replaced with others from a different supplier manufactured by a different method. We call this concept "Total MPD Management."
\end{abstract}

Index Terms-EAF, encircled flux, encircled angular flux, EMD, EF, equilibrium mode distribution, HPCF, MMF, multimode, optical fibers, overfilled launch, offset launch, plastic optical fiber, POF, PCF, restricted launch, step index, short reach, very short reach.

\section{INTRODUCTION}

$\mathbf{L}$ OW cost multimode optical fibers (MMFs) are being introduced for short distance communication applications,

Manuscript received August 18, 2015; revised December 28, 2015 and November 16, 2015; accepted December 30, 2015. Date of current version February 5, 2016. This work was supported in part by "International standardization and dissemination project for high-speed communication network performance over large core multimode optical fiber-Technology integration for O-GEAR: Optical Gigabit Ethernet for Automotive aRchitecture" Project of the Ministry of Economy, Trade and Industry (METI) in Japan.

M. Kagami, A. Kawasaki, M. Yonemura, and M. Nakai are with the Toyota Central R\&D Labs., Inc., Nagakute, Aichi 480r-1192, Japan (e-mail: kagami@mosk.tytlabs.co.jp; akari@mosk.tytlabs.co.jp; yonemura@mosk. tytlabs.co.jp; m-nakai@mosk.tytlabs.co.jp).

P. V. Mena is with the Synopsys, Inc., Ossining, NY 10562 USA (e-mail: pablo@synopsys.com).

D. R. Selviah is with the Electronic and Electrical Engineering Department, University College London, UCL, London (e-mail: d.selviah@ucl.ac.uk).

Color versions of one or more of the figures in this paper are available online at http://ieeexplore.ieee.org.

Digital Object Identifier 10.1109/JLT.2016.2516644 such as those used in work premises, at home, in vehicles and for rack-to-rack communications typically less than $50 \mathrm{~m}$. Recently, there has been a trend to substantially raise the communication data rate and system stability by controlling the launched mode conditions to the MMF [1]-[2]. In these short reach communication areas, large core and high NA step-index multimode optical fibers (SI-MMFs), such as hard polymer clad fiber (HPCF) and plastic optical fiber (POF), have been implemented [3]-[5] because the bandwidth degradation due to mode dispersion of these fibers is limited. HPCF is also known as Polymer Clad Silica, PCS or Hard Polymer Clad Silica, HPCS. Particularly, since SI-MMFs have large lateral and angular misalignment tolerances [6] and consequently lower connector and, hence, system cost, network systems using these fibers are expected to increase in number. The latest research affirms that multi-level coding [7] and mode-division multiplexing [8] technologies will enable systems employing SI-MMFs, under limited mode launch conditions, to achieve beyond one gigabit communication rates. However, since the modal power distribution (MPD) in SI-MMFs gradually changes during propagation as a result of scattering in the core and at the boundary with the cladding, fiber bending and the insertion of passive components, an accurate measurement method and a form of representation for the MPD are needed. It is well known that the main transmission characteristics of a multimode optical component, i.e., the insertion loss, dispersion and bandwidth [9]-[12], depend largely on the MPD at launch. As the spread of short reach communications employing SI-MMFs advance, the need to specify a MPD measurement method and a way of representing it, which can serve as an international standard, is becoming imperative. This will enable network designers to put the MPD in a specification and will encourage the proliferation of multi-vendor systems. In particular, in the automotive industry [13] there is a trend towards auto-drive applications where the communication speed required for the sensor network involving high-resolution and high-frame-rate image sensors is rapidly increasing, about tenfold every five years. Many manufacturers currently measure multimode components and fibers using their own different and incompatible methods and this needs to be remedied by introducing international standardization.

Another typical MMF, graded-index multimode optical fiber (GI-MMF), is commonly used in longer and faster data transmission applications over distances typically less than several hundred meters. For MPD measurements of GI-MMF, three simple methods have been used [14], [15]; one is the CPR (Coupled Power Ratio) method [9], another is the DMD (Differential 

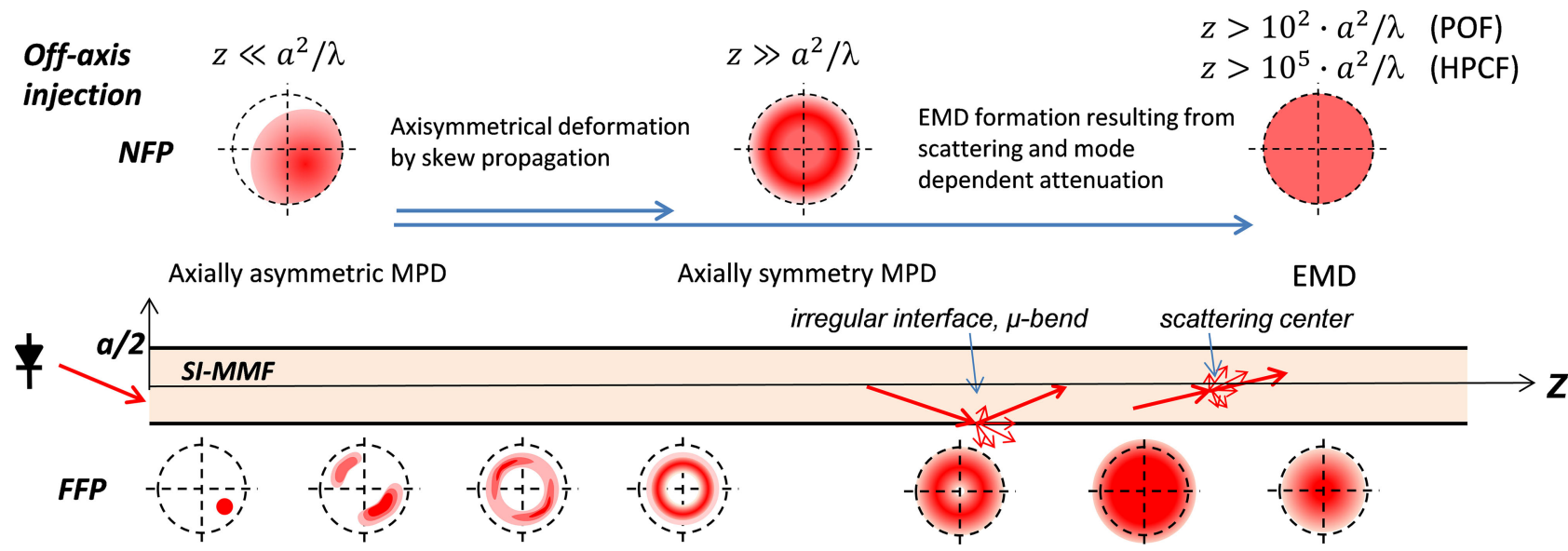

Fig. 1. Conceptual diagram of MPD transformation and its principal causes along a SI-MMF after a narrow beam is injected at an angle to and laterally displaced from the fiber axis. The upper and lower cross-sectional illustrations show the NFP and FFP cross section respectively, when the fiber is cut at each stage.

mode delay) method [16]. CPR shows the ratio of the transmission of a low order mode component to transmission of all of the generated modes using a single mode fiber. The DMD method enables one to deduce the rough output MPD from a mode dispersion measurement of relatively long fiber when the launched MPD is known. In recent years, a simple and highly reproducible MPD measurement method, the EF (Encircled Flux) method, which infers the MPD from the near field pattern (NFP) at the end face of a GI-MMF, has been proposed [17] and this has been standardized internationally by the IEC [18]. The EF method uses the characteristic that higher-order modes take an outer optical path in the core due to the index gradient. However, this method is not applicable for SI-MMFs, since all modes (or rays) take the same space within the uniform index core crosssection. The best of these three methods is the EF method because of its quantitative performance, and its applicability to 10 $\mathrm{Gb} / \mathrm{s}$ and higher bit rate optical transmitters [17]. Furthermore, although the limited phase space [19], [20] has often been used at launch, the allowable variations need to be defined using a reproducible form of notation.

For MPD measurements of SI-MMFs, the loss test measurement method [19] defines launch conditions that have the same spot size and NA as the diameter of the fiber core and the maximum NA, respectively. As described above, there is neither an accurate MPD measurement method nor a strict form of representation that takes the asymmetry of the MPD into consideration for SI-MMFs.

Research aiming to standardize the launch conditions [15], [21]-[25] has concluded that the measurement of fiber attenuation is more sensitive to variations in the NA than it is to the $\mathrm{CD}$ [6] so that it is more important to define a new launch condition standard based on angular power distribution. Since all the propagating modes couple and transmit with uniformity inside the whole core cross-section of the SI-MMF, the relation between the MPD and NFP disappears. Therefore, a straightforward representation of the MPD calculated from FFP (far field pattern) measurements is necessary for the fiber. We have been working to define and to standardize this new EAF (Encircled Angular Flux) for a SI-MMF [26], [27]. This paper defines the theoretical relationship between EF and EAF in polar and Cartesian coordinates in detail and shows experimental EAF data. Both EF and EAF represent the relative intensity of the transmitted power in a limited range with respect to the whole transmitted power, but the EF is calculated from a NFP and the EAF is calculated from a FFP. The Modal Power Distribution, MPD, which is the power distribution over modes, is neither the NFP nor the far field pattern but can be inferred from them. By using the EAF representation, we demonstrated for the first time a peculiar mode transfer phenomenon in a SI-MMF, the behavior of the MPD as it progresses towards an equilibrium mode distribution (EMD) and the possibility of a definition of an EAF-template for SI-MMF like the EF-template [18] defined for the insertion loss measurement of GI-MMF. Recent research has shown that by using mandrel winding a standard EAF can be generated which is independent of the source lateral and angular power distribution characteristics [22] and so provides a readily reproducible standard for launch conditions.

\section{MPD TRANSITION IN A SI-MMF}

Since the early 1970s, many researchers have reported both experimental and theoretical mode transition phenomena along a SI-MMF [28]-[30]. Generally, in an optical fiber, there are many scattering centers due to irregular structures at the core/cladding boundary and refractive index variations in the core. At those centers, the transmitted light is always subject to weak mode conversion due mainly to forward scattering. This conversion continues until the transmitted MPD becomes the fiber's inherent Equilibrium Mode Distribution EMD. Fig. 1 shows the general concept of MPD transformation. When a narrow beam of light is launched at an angle to and laterally displaced from the SI-MMF fiber axis, we found from our many experiments characterizing the NFP that the NFP gradually spreads into a concentric pattern and becomes axisymmetric in the Fraunhofer region. After that, the NFP changes from being axisymmetric to a uniform pattern. This phenomenon appears prominently in a larger scattering fiber and in tight spooled fiber [31], [32]. Table I shows the typical specifications for HPCF and POF, 
TABLE I

TYPICAL SI-MMFS

\begin{tabular}{lcc}
\hline \hline Optical fiber & HPCF & POF \\
\hline IEC classification & Category A3e & Category A4a.2 \\
& IEC 60793-2-30 & IEC 60793-2-40 \\
Core diameter $(\mu \mathrm{m})$ & $200 \pm 4$ & $990 \pm 85$ \\
NA & $0.37 \pm 0.02$ & $0.485 \pm 0.045$ \\
Typical wavelength $(\mathrm{nm})$ & $770-870$ & $500-690$ \\
\hline \hline
\end{tabular}

both representative SI-MMFs. The lengths of fiber required to reach their inherent EMDs have been found by the authors to be $z>10^{2} \cdot a^{2} / \lambda$ and $z>10^{5} \cdot a^{2} / \lambda$ for POF and HPCF, respectively, where, $a$ is the core radius and $\lambda$ is the wavelength. This two orders of magnitude difference is mainly due to the transparencies and scattering coefficients of the core materials, PMMA and silica respectively. The details of how the MPD changes along a fiber will be discussed in Section IV, but a schematic illustration of the transition of the FFP image, which is obtained when a fiber is cut at various points, is shown in the lower part of Fig. 1. As for the NFP, in the Fraunhofer region, the profile of the FFP becomes axisymmetric and approaches equilibrium [26]. There are many studies dealing with this FFP transition process, especially with a view towards finding modeconversion coefficients and differential mode attenuation [33]. However, there is no reproducible and useful visual form for representing the MPD for the network designer, who needs to construct a system taking into account the dependence of the incident MPD on the first optical components performance and its outgoing MPD on the performance of the subsequent optical component. This is particularly important, in the case of very short reach transmission, especially less than several meters for example, in which both the NFP and FFP along an optical fiber at various positions are non-axisymmetric and change significantly along the length of an optical fiber. The fibers bandwidth dependence on MPD is very sensitive [9]-[12], so an accurate method for measuring and representing MPDs is required. Therefore, to meet these requirements, this paper reports a new method for accurately measuring and visually representing the MPD.

\section{SI-MMFs AND Multimode COMPONENTS}

As an example of a multimode component, the insertion loss dependence of a WDM (wavelength division multiplexer) element in a bidirectional optical transceiver module [34] developed for SI-MMF networks has a dependence on the MPD launch conditions as shown in Fig. 2. A 3-D polymer optical waveguide with a circular cross section, connected to a lead-in SI-POF, has a T-shape branched waveguide, with a WDM filter tilted at an angle of $45^{\circ}$. The waveguides are automatically formed using LISW (Light-Induced Self-Written) technology [35]. In this element, the choice of launch mode conditions affects the measured reflection and transmission properties of the WDM filter, and the coupling properties between the POF and the waveguide. Fig. 2(c) shows the dependence of the insertion loss on the SI-POF lead fiber length (L) for the launch conditions of over filled launch (OFL) and restricted lower order

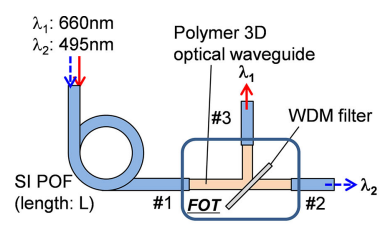

(a)

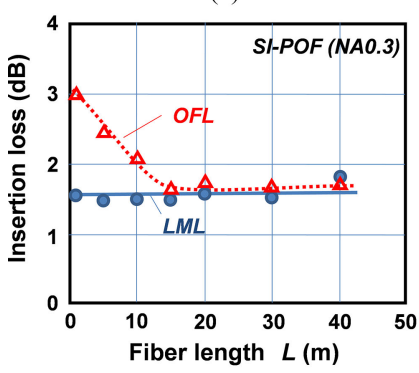

(c)

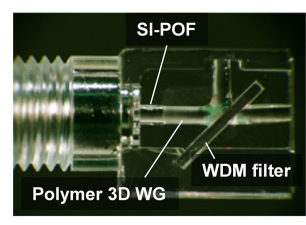

(b)

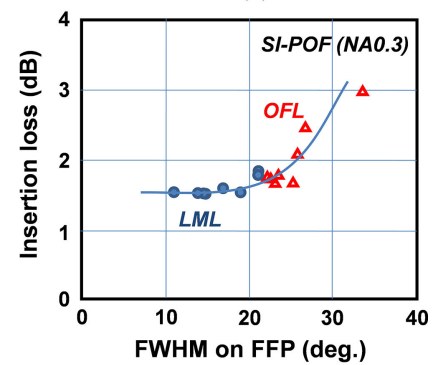

(d)
Fig. 2. Example of MPD dependence on the insertion loss of a multimode waveguide WDM device, (a) tested WDM element structure, (b) WDM element fabricated by LISW (light-induced self-written) waveguide technology, (c) dependence of insertion loss (\#1 to \#2) on lead fiber (smaller NA POF) length and launch condition, (d) dependence of insertion loss on FWHM of FFP of input optical launch conditions to the waveguide component. The increasing loss is due to the WDM filter and not the waveguide NA. These results include a measurement error.

mode group launch (LML) through port \#1. It can be seen that the insertion loss falls as the lead-in fiber length increases up to $15 \mathrm{~m}$ in the case of OFL, which uses a LED butt-coupled to the POF. On the other hand, the insertion loss variation versus L is within measurement accuracy in the case of LML. When the Insertion loss is instead plotted as a function of the FWHM of the FFP of the light distribution launched into the lead-in fiber, extracted from a one-dimensional (1-D) FFP taken from the plane of the two-dimensional (2-D) FFP at the input face of the polymer waveguide, we find the insertion loss remains flat to within measurement accuracy up to about $19^{\circ}$ after which it increases with the FWHM, as shown in Fig. 2(d). Since the waveguide NA is 0.42 and slightly smaller than that of POF (smaller NA, Mitsubishi ESKA-MEGA) [34], it is considered that this loss increase comes from a transmittance dependence on incident angle of the WDM filter. It can be seen from these two graphs that the insertion loss can vary over a range of about $1.5 \mathrm{~dB}$ depending on the angular divergence of the launched light at the end of the lead-in fiber and the length of the leadin fiber. The large variation in insertion loss for the OFL case means a large MPD transition to the EMD, occurs over $15 \mathrm{~m}$ of SI-MMF. This MPD transition is certain to occur in all kinds of MMF. Therefore, in performing characterization, it is important to describe the MPD in a reproducible way.

\section{MPD TRANSITION IN SI-MMF}

\section{A. MPD Launch Conditions}

Next, we investigated the MPD transition along the SI-MMF. We measured the FFPs of outgoing beams after transmission through from 3 to $3000 \mathrm{~m}$ of HPCF (OFS, HPCF-M0200T, for properties refer to Table I). The FFP measurement apparatus used (Hamamatsu Photonics, LEPAS-11) consists of an $\mathrm{f}-\theta$ lens 


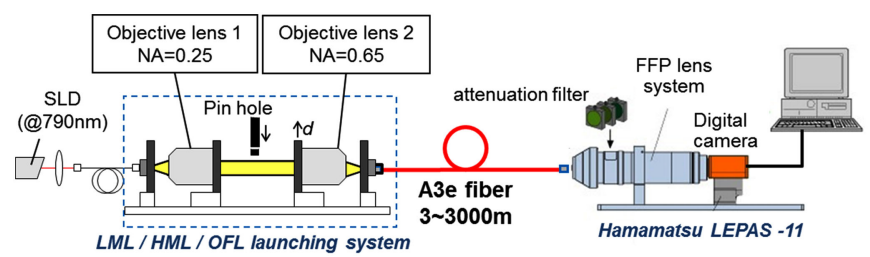

(a)

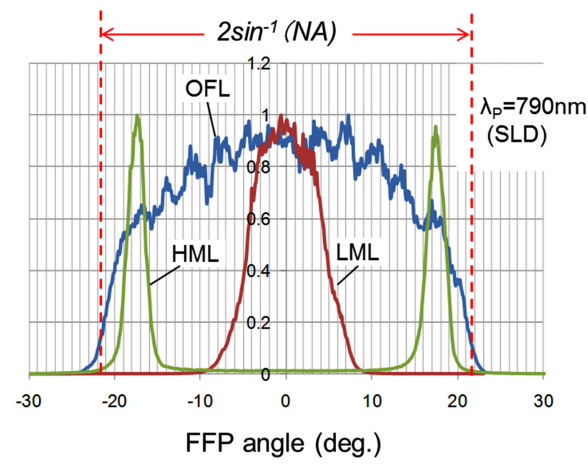

(b)

Fig. 3. Mode group launches (OFL, LML, HML) and FFP observation system, (a) Launch experiment comprising $790 \mathrm{~nm}$ SLD (Super Luminescent Diode) and objective lens, and FFP measurement system, (b) 1-D FFPs for each MPD launch condition measured on the outgoing beam from $6 \mathrm{~m}$ of HPCF. All these 1-D profiles were extracted from a plane normal to the optical axis through the centroid of the 2-D FFP, $\theta \mathrm{x}=0, \theta \mathrm{y}=-30^{\circ}$ to $+30^{\circ}$, These angles are defined in Fig. 7. The vertical red dashed lines indicate the limits of the theoretical NA calculated from the core and cladding refractive indices.

and a highly sensitive imaging system. The optical system for OFL of the HPCF is shown in Fig. 3(a). A pinhole aperture with a diameter of $0.2 \mathrm{~mm}$ is inserted on the axis of the collimated beam to create a LML. In addition, an objective lens 2 can be translated transversely normal to the optical axis by $\sim 1.5 \mathrm{~mm}$, to give an offset launch without incurring additional loss as this amount of offset meets the requirement for the maximum acceptance angle of the fiber when a restricted higher-order-mode group is launched (HML). The FFPs after $6 \mathrm{~m}$ transmitted under these three launch conditions (OFL, LML, HML) are plotted in Fig. 3(b). Fig. 3(b) shows the whole FFP light distribution due to light coupled into both the core and the cladding including both bound modes and any remaining evanescent radiation modes which have not yet radiated all away. One can see the cladding mode observed in the OFL conditions in the area outside the theoretical NA indicated by the red dashed lines. Furthermore, one can also see that the limited mode group launches are well done because the beam divergence is small for both the LML and HML conditions.

Next, the MPD transitions along the long HPCF under the three launch conditions are plotted in Fig. 4 as a function of increasing fiber length. In the case of LML, the higher-order components gradually increase as a result of internal scattering in the core, scattering at the interface between the core and the cladding, and gentle fiber bends by spooling on a $47 \mathrm{~cm}$ diameter drum. In the case of HML, the MPD at launch has a peak at about $17^{\circ}$ and a width of $\pm 1.5^{\circ}$. Only the width increases while the peak angle remains the same for the same reasons as the LML case. Since the higher-order modes are more susceptible to both boundary imperfections and attenuation due to the longer optical

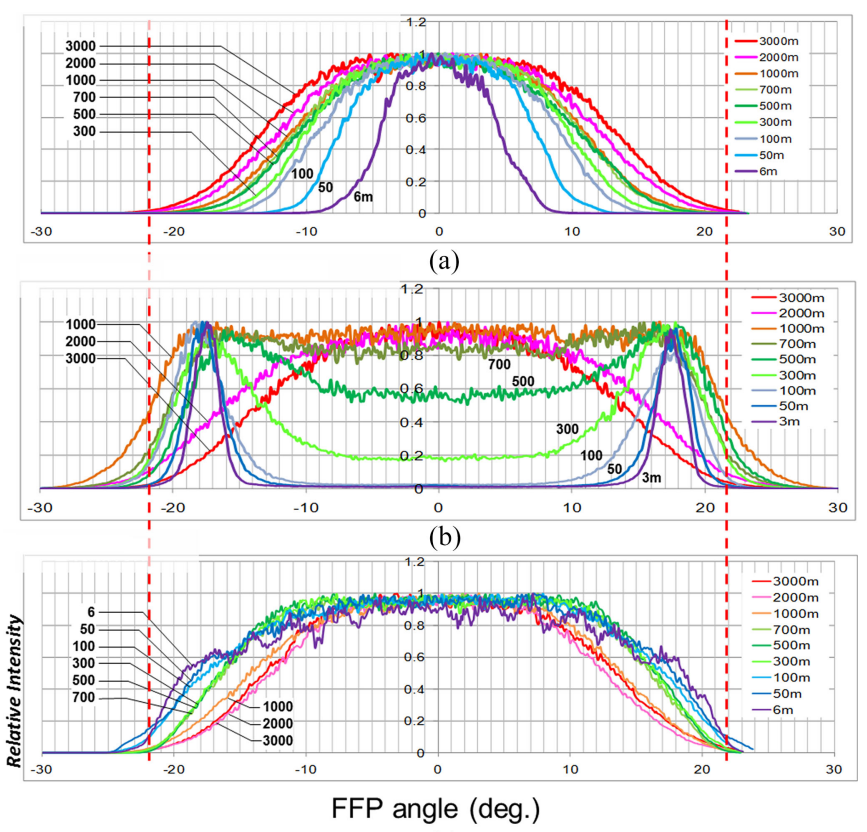

(c)

Fig. 4. MPD transition under three different launch conditions, (a) LML, (b) HML, and (c) OFL. All these 1-D profiles were extracted from a plane normal to the optical axis through the centroid of the 2-D FFP, $\theta \mathrm{x}=0, \theta \mathrm{y}=$ $-30^{\circ}$ to $+30^{\circ}$, These angles are defined in Fig. 7. The vertical red dashed lines indicate the limits of the theoretical NA calculated from the core and cladding refractive indices.

path, the lower-order mode components gradually increase. As a result, a "top-hat" shaped MPD is formed at a fiber length of around $\mathrm{L} \sim 1000 \mathrm{~m}$. Intriguingly, the evanescent tails are most significant at this length. Beyond this, the higher order modes continue to be further attenuated, and the top hat MPD approaches a Gaussian shape which is the EMD for this fiber. Meanwhile, although the Gaussian like MPD is maintained with OFL, its width varies towards that of the EMD because of the large attenuation loss due to the cladding and the higher order mode components. From these radically different MPD launch conditions, it is considered that this fiber converts the input launch to an EMD regardless of the MPD launch conditions if the fiber is more than $2000 \mathrm{~m}$ in length. Details are showed in Section IV-D.2.

\section{B. MPD Dependence on Connector Loss}

Next, we conducted a detailed study of the effect of the insertion loss of an optical component due to a variation in MPD. Using just the LML and HML launch conditions, the MPD varies significantly along the fiber. We calculated the insertion loss of a PN optical connector [36] placed at various points along an HPCF optical fiber using LightTools software. We generated 100,000 rays and confirmed it is saturated within $0.1 \%$ accuracy. The results are shown in Fig. 5. The insertion loss was calculated for the worst-case lateral offset, with conventionally used parameters; lateral offset of $40 \mu \mathrm{m}$; air gap of $100 \mu \mathrm{m}$; tilt of the optical axis $0.5^{\circ}$, using the actual measured MPDs shown in Fig. 4. Remarkably, the insertion loss was a maximum at $\mathrm{L} \sim 1000 \mathrm{~m}$ for the HML launch condition. This means that when a higher order mode group was launched by tilting of the 


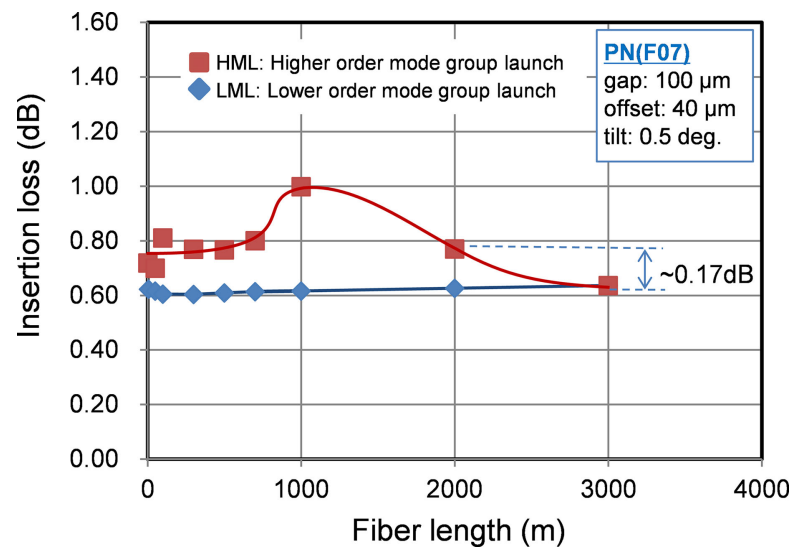

Fig. 5. Simulated Insertion loss of a PN (F07) connector inserted in a MMF. Insertion losses under the worst connection case are calculated using the actual measured MPD for each fiber length.

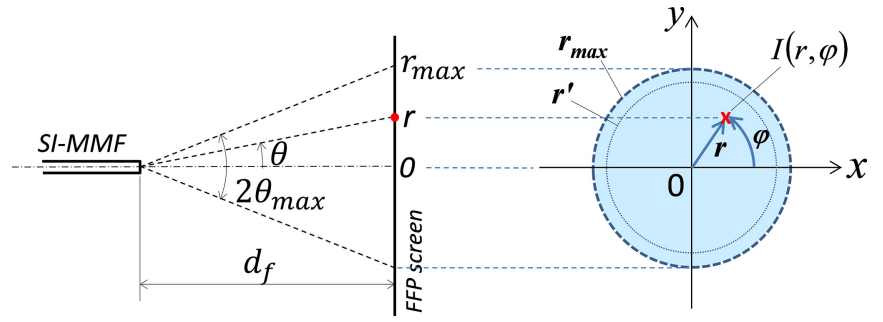

(a)

(b)

Fig. 6. Coordinate system for FFP measurements, (a) Measurement parameters in the meridional plane including the measurement point $r$ and the optical fiber axis. The maximum angle of radiation from the SI-MMF is $2 \theta_{\max }$. Note that, $\theta$ is not a projection angle but an angle in the meridional plane, (b) polar coordinate system $(r, \varphi)$ on the FFP screen.

optical axis, for example, the propagation loss in the MMF at this distance was greater than that near the incident face. This is attributable to the evanescent tails of the core modes extending into the cladding that reach a maximum at $\mathrm{L} \sim 1000 \mathrm{~m}$, as shown in Fig. 4 (b). There are variations in insertion loss from 0.2 to $0.4 \mathrm{~dB}$ for fiber lengths of $1000 \mathrm{~m}$ or less. Since the evanescent tails are sensitive to coupling loss, we need, during design, to consider the launching conditions in order to avoid generation of them. The HML and LML curves in Fig. 5 remain separate and only come together at a fiber length of $3000 \mathrm{~m}$. Therefore this work shows that the MPD does not reach the Equilibrium Modal Power Distribution, EMD, until $3000 \mathrm{~m}$ and that $2000 \mathrm{~m}$ is not a sufficient length. This result is also in agreement with the results we present later in this paper for EAF.

\section{Reproducible MPD Representation}

1) Definition of EAF in Polar Coordinates: Defining EAF in a similar manner to the definition of EF for GI-MMFs is important in order to gain universal acceptance. So we define the EAF as an EF on a FFP screen not a fiber end-face [26], [27], as shown in Fig. 6. Similar to the EF representation of MPD, we define the EAF as the relative intensity of an arbitrary radiation flux, within a given cone angle, to the total flux, assuming a maximum radiation cone with a plane angle of $2 \theta_{\max }$. The positional relationships between the end face of a SI-MMF and a screen located sufficiently far from the end face $\left(d_{f} \gg a^{2} / \lambda\right)$, and the polar $(r, \varphi)$ and Cartesian $(x, y)$ coordinate systems on the screen are shown in Fig. 6(a) and (b), respectively, where, $a$ and $\lambda$ are the diameter of the core and the wavelength, respectively. Light radiated from the fiber end is projected onto the screen and we can observe its intensity distribution $I(r, \varphi)$ as a far-field-pattern. If the distribution has axial symmetry $I(r, \varphi)=\left.I(x)\right|_{\varphi=0}$, the assumed NFP on the screen can be described by eq. (1) using the EF definition [17]

$$
E F\left(r^{\prime}\right)=\frac{\int_{0}^{r^{\prime}} I(x) \cdot x \cdot d x}{\int_{0}^{r_{\max }} I(x) \cdot x \cdot d x} .
$$

If the distribution has non-axial symmetry, the relative ratio of the flux passing within a circle of radius $r^{\prime}$ to that of the whole field of radius $r_{\max }$ can be expressed in polar coordinates by,

$$
E F\left(r^{\prime}\right)=\frac{\int_{0}^{2 \pi} \int_{0}^{r^{\prime}} I(r, \varphi) \cdot r \cdot d r \cdot d \varphi}{\int_{0}^{2 \pi} \int_{0}^{r_{\max }} I(r, \varphi) \cdot r \cdot d r \cdot d \varphi}
$$

where, $I(r, \varphi)$ is the light intensity at point $(r, \varphi)$, and its units are $m^{-2}$.

Next, we convert the NFP obtained from eq. (2) into the FFP of a radiated beam from the fiber end. The polar coordinate system on the screen is related to the distance from the fiber end to the screen $d_{f}$ and radiation angle $\theta$, and this relationship is described by

$$
r=d_{f} \cdot \tan (\theta) .
$$

Hence, $\theta$ and $\varphi$ are independent, and by using eq. (3) and its differential form, we can obtain the following relationship

$$
r \cdot \mathrm{d} r=d_{f}^{2} \cdot \sin (\theta) / \cos ^{3}(\theta) \cdot d \theta .
$$

Substituting this into eq. (2), we obtain $\operatorname{EAF}\left(\theta^{\prime}\right)$, the relative flux within a cone of half angle $\theta^{\prime}$, as follows:

$$
\operatorname{EAF}\left(\theta^{\prime}\right)=\frac{\int_{0}^{2 \pi} \int_{0}^{\theta^{\prime}} I(\theta, \varphi) \cdot \frac{\sin (\theta)}{\cos ^{3}(\theta)} \cdot d \theta d \varphi}{\int_{0}^{2 \pi} \int_{0}^{\theta_{\max }} I(\theta, \varphi) \cdot \frac{\sin (\theta)}{\cos ^{3}(\theta)} \cdot d \theta d \varphi}
$$

where, EAF is defined as EF with taking account of projection angle $\theta$.

2) Definition of EAF in Cartesian coordinates: Equation (5) is mathematically correct but may be inconvenient for some who want to calculate the EAF using the commercially available FFP system with Cartesian coordinate output format. In this system, far-field measurement gives the optical intensity as a function of the angles $\theta_{x}$ and $\theta_{y}$, i.e., $I\left(\theta_{x}, \theta_{y}\right)$, where Fig. 7 illustrates the relationships between $\theta_{x}, \theta_{y}$, the far-field distance $d_{f}$ and the $(x, y)$ coordinates in the far-field plane. We also show the polar coordinates $(r, \varphi)$ in the far-field plane, and the relationship between $\theta, d_{f}$ and $r$,

$$
\begin{aligned}
x & =d_{f} \cdot \tan \theta_{x} \\
y & =d_{f} \cdot \tan \theta_{y} \\
r & =d_{f} \cdot \tan \theta \\
r^{2} & =x^{2}+y^{2} .
\end{aligned}
$$




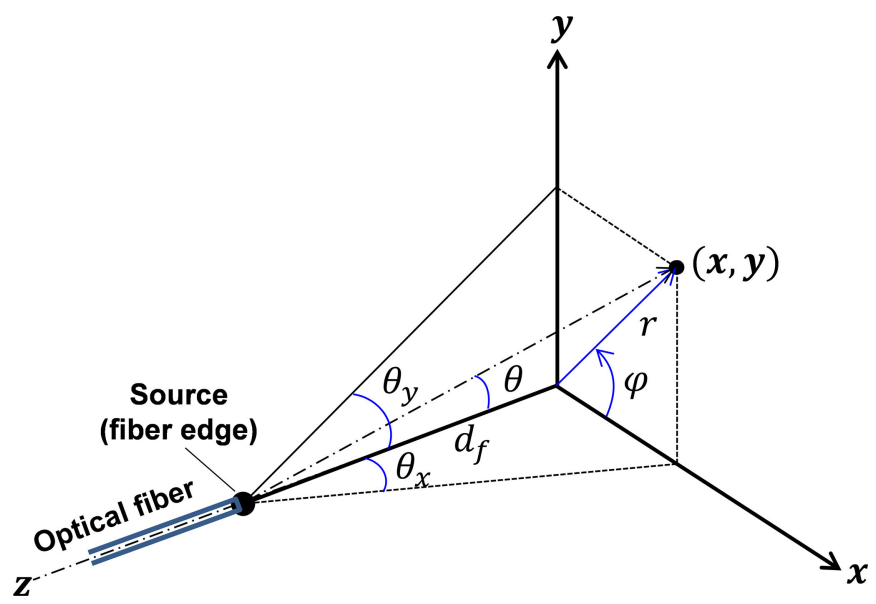

Fig. 7. Cartesian coordinates in far-field measurement. Plane $x-y$ is the far-field plane.

From these equations we also obtain

$$
\theta=\arctan \left(\sqrt{\tan ^{2} \theta_{x}+\tan ^{2} \theta_{y}}\right) .
$$

We begin by converting equation (2) expressed in polar coordinates into an equation in Cartesian coordinates; we obtain:

$$
E F\left(r^{\prime}\right)=\frac{\int_{-r}^{r} \int_{-\sqrt{r^{2}-y^{2}}}^{\sqrt{r^{2}-y^{2}}} I(x, y) \cdot d x \cdot d y}{\int_{-r_{\max }}^{r_{\max }} \int_{-\sqrt{r_{\max }^{2}-y^{2}}}^{\sqrt{r_{\text {max }}^{2}-y^{2}}} I(x, y) \cdot d x \cdot d y} .
$$

Using equations (6) and (7), we obtain:

$$
d x=\frac{d_{f}}{\cos ^{2} \theta_{x}} \cdot d \theta_{x}, \quad d y=\frac{d_{f}}{\cos ^{2} \theta_{y}} \cdot d \theta_{y} .
$$

Substituting these equations and eq. (10) into the encircled flux equation (11), with the appropriate conversion of the limits of integration, we obtain the following equation for calculating the EAF:

$$
\begin{aligned}
& \operatorname{EAF}(\theta)= \\
& \frac{\int_{-\theta}^{\theta} \int_{-\operatorname{atan}\left(\sqrt{\tan ^{2} \theta-\tan ^{2} \theta_{y}}\right)}^{\operatorname{atan}\left(\sqrt{\tan ^{2} \theta-\tan ^{2} \theta_{y}}\right)} \frac{I\left(\theta_{x}, \theta_{y}\right)}{\cos ^{2} \theta_{x} \cdot \cos ^{2} \theta_{y}} \cdot d \theta_{x} \cdot d \theta_{y}}{\int_{-\theta_{\max }}^{\theta_{\max }} \int_{-\operatorname{atan}\left(\sqrt{\tan ^{2} \theta_{\max }-\tan ^{2} \theta_{y}}\right)}^{\operatorname{atan}\left(\sqrt{\tan ^{2} \theta_{\max }-\tan ^{2} \theta_{y}}\right)} \frac{I\left(\theta_{x}, \theta_{y}\right)}{\cos ^{2} \theta_{x} \cdot \cos ^{2} \theta_{y}} \cdot d \theta_{x} \cdot d \theta_{y}}
\end{aligned}
$$

where $\theta_{\max }$ is the maximum radiation angle, and given the light propagating at angles more than the theoretical NA calculated from the core and cladding refractive indices, shown in Figs. 3 and 4 , it is best to set the range of integration so that $\theta_{\max } \gg N A$ in order to obtain an accurate calculation.

\section{MPD Transitions Described in the EAF System}

1) MPD Launch Conditions: For the actual measurements, we chose the measurement range of $\theta_{\max }=30^{\circ}=1.35 \cdot \mathrm{NA}$ for the HPCF optical fiber (NA 0.37, see Table I). We confirmed that this measurement range could take account of complete

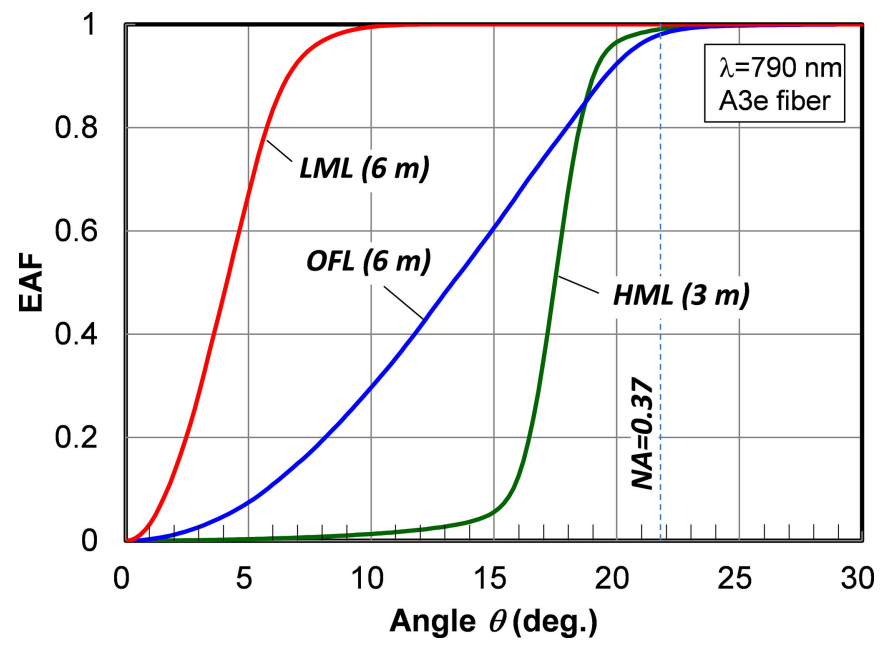

Fig. 8. Examples of EAF notation using 2-D FFP data. Note that in Fig. 3, only 1-D FFP data was used. Calculated from MPDs after passing through 6, 3 and $6 \mathrm{~m}$ of $\mathrm{A} 3 \mathrm{e}$ MMF for the LML, HML and OFL launch conditions, respectively.

evanescent tails under HML and OFL conditions, as shown in Fig. 4. From Fig. 4(b), the evanescent tails extend to 29 degrees while the maximum radiation angle, calculated from the theoretical NA of HPCF, is $21.7^{\circ}$. So, 29/21.7 = 1.34. For the typical launch modes we chose the three MPDs shown in Fig. 3(b). These have been re-plotted in Fig. 8 using the EAF representation defined by eq. (13). For the LML condition, we find most radiated power is concentrated within $\theta \leq 10^{\circ}$. In contrast, for the HML condition, hardly any radiated power is observed within $\theta \leq 15^{\circ}$. Because the OFL condition includes some cladding modes or evanescent tails, even in the region of $\theta>21.7^{\circ}$ a certain level of transmitted power is observed. In all cases, if the calculation covers the range of $\theta_{\max }=1.35 \cdot \mathrm{NA}$, the whole transmitted power is considered in the calculation. Because of the integral representation, the EAF gives smooth curves compared with the conventional method of representing MPD by the extracted intensity profile on the plane normal to the optical axis through the far field intensity centroid described in Fig. 3 (b). Particularly, the EAF curves help to accurately determine the MPD which is important to effectively design networks with the required performance.

2) EMD Measurement: In actual FFP measurements, we used the f- $\theta$ lens system (LEPAS 11 , Hamamatsu) and an imaging device. The image was corrected to remove background light [27] usually $0.5 \%$ or less of the maximum intensity and to allow for the tilt of the optical axis.

Almost all of the MPDs would fall within the range between the LML and HML curves shown in Fig. 8. Therefore, we measured the transition of the MPD along a SI-MMF under the launch conditions of these two radically different MPDs. Flat polished SI-MMFs (IEC category; A3e) with lengths from 3 to $3000 \mathrm{~m}$ were prepared and the EAF measured at wavelengths of 790 and $850 \mathrm{~nm}$, as shown in Fig. 9. At both wavelengths, the MPDs approach the same EAF curves toward $3000 \mathrm{~m}$ regardless of the launch conditions. These EAF curves are the fiber's intrinsic EMD for each wavelength. By comparison of 


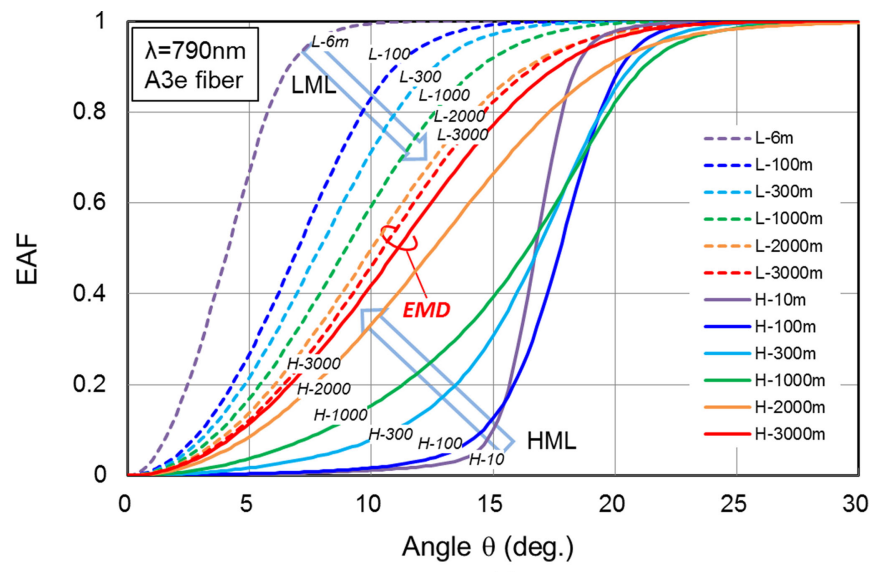

(a)

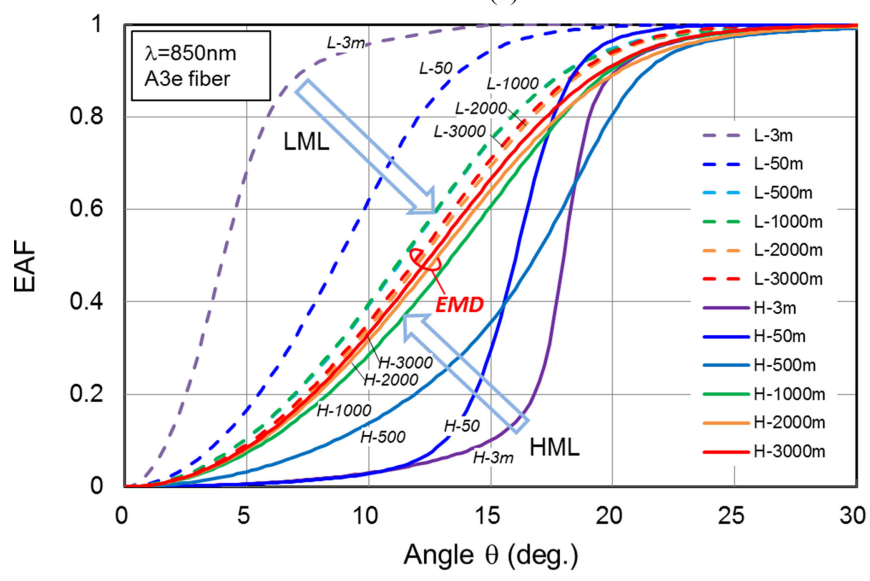

(b)

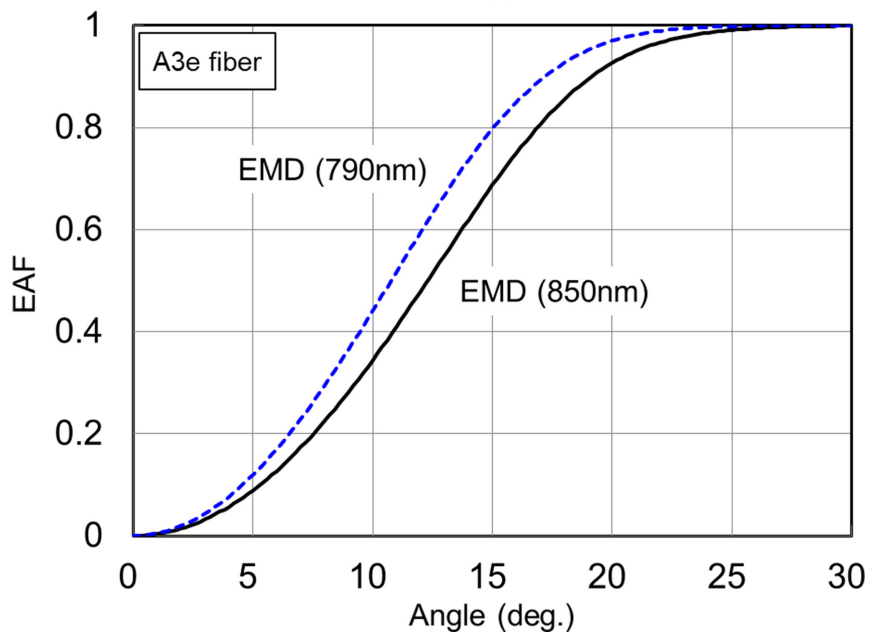

(c)

Fig. 9. EAF of various lengths of A3e SI-MMF (HPCF) for LML conditions and HML conditions, at wavelength (a) $790 \mathrm{~nm}$, (b) $850 \mathrm{~nm}$. (c) EMDs comparison for wavelength of 790 and $850 \mathrm{~nm}$.

the EMDs for each wavelength, as shown in Fig. 9(c), we find the amount of variation towards EMD per unit length for $790 \mathrm{~nm}$ is smaller because there is less attenuation and scattering. As a result, the EAF curves for $850 \mathrm{~nm}$ at $2000 \mathrm{~m}$ are closer to the EMD curves. From the above observations, we consider that the EMD at $850 \mathrm{~nm}$ has shifted towards a higher-order mode due to the lower MDA (Mode-Dependent Attenuation) [37] impact.

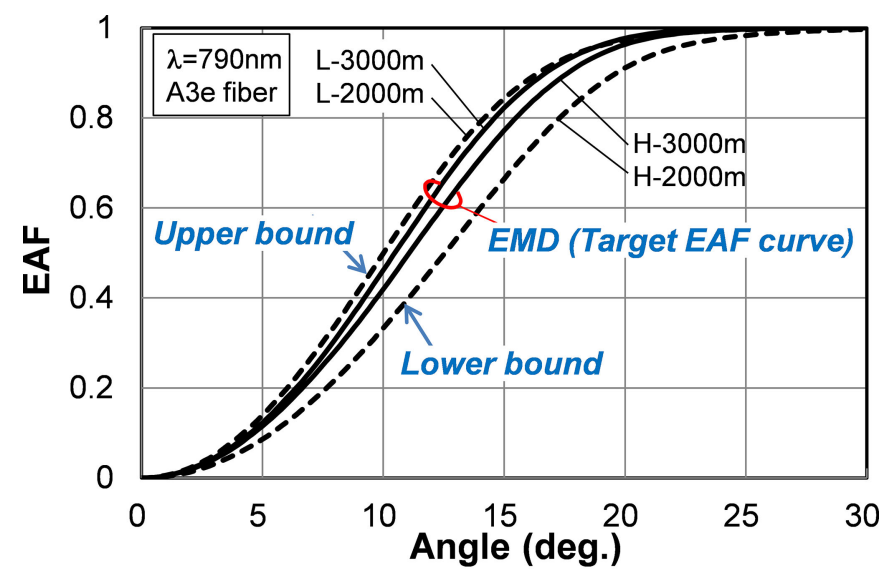

(a)

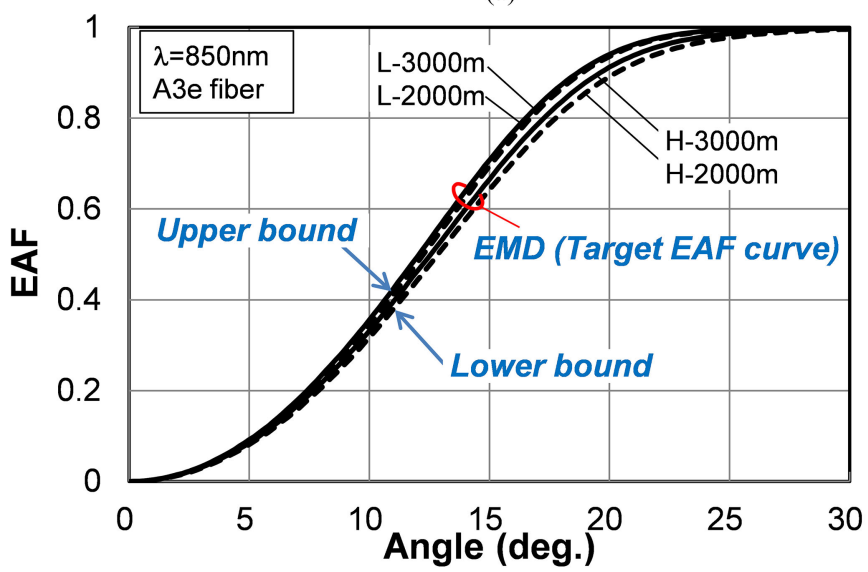

(b)

Fig. 10. Examples of EAF templates for attenuation measurements in A3e MMF, wavelength of (a) $790 \mathrm{~nm}$, (b) $850 \mathrm{~nm}$.

3) MPD Templates for Attenuation Measurements: In this section "attenuation" refers to the insertion losses due to optical components inserted in the fiber, the coupling loss into an inline optical component and out from it into an optical fiber and a distributed constant for the propagation loss $(\mathrm{dB} / \mathrm{m})$ in the fiber. The objective of the launch MPD template is to limit the attenuation variance to a specified percentage, $\pm s$, (e.g., $10 \%$ ) of the target EMD or $\pm \mathrm{S} \mathrm{dB}$, whichever is the larger. $\mathrm{S}$ is called the tolerance threshold and varies depending on the MMF parameters [38]. There is no $\mathrm{S}$ regulation for $\mathrm{A} 3 \mathrm{e}$ fiber, but we use $S$ of $0.2 \mathrm{~dB}$, which is a commonly used value for low loss devices like the optical connectors used in this experiment. As can be seen from the example shown in Fig. 5 of the insertion loss for a PN connector, if an attenuation loss measurement of a passive optical component connected to $2000 \mathrm{~m}$ or more of lead fiber is carried out, reproducible results with variations less than $0.2 \mathrm{~dB}$, regardless of the MPD launch conditions, can be obtained. The mode dependency of the attenuation of passive devices varies from structure to structure, but we opted for this classic component, the most widely used connector for A3e MMF. Therefore, when launching a MPD by LML or HML within the "EAF template", we can perform an attenuation measurement after passing through $2000 \mathrm{~m}$ of fiber with $0.2 \mathrm{~dB}$ or $10 \%$ accuracy. The EAF template examples for wavelengths 
of 790 and $850 \mathrm{~nm}$ are shown in Fig. 10. The solid lines in the figures are the EAFs after passing through $3000 \mathrm{~m}$ of fiber, and because these lines gravitate towards the same curve, we can say these are the EMDs for each wavelength. When an optical fiber is wound around a bobbin in multiple layers, some random tension is applied to the fiber. Some shift in the EAF curve is frequently observed in a long length optical fiber (upper bound for the LML launch through a $3000 \mathrm{~m}$ fiber in Fig. 10(b)) as a result of this tension. The dashed curves in Fig. 10 correspond to $\pm \mathrm{S} \mathrm{dB}$ where $\mathrm{S}=0.2 \mathrm{~dB}$ (see Fig. 5). In this case, the EAF curve at $\mathrm{L} \sim 2000 \mathrm{~m}$ means a tolerance of $0.2 \mathrm{~dB}$. This can only apply for a F07 connector. We consider these EMD curves to be the "target EAFs." These results are in agreement with the completely different earlier insertion loss measurements of Fig. 5 where we showed that a fiber length of at least $3000 \mathrm{~m}$ is required in order to achieve the EMD and that $2000 \mathrm{~m}$ length is insufficient for this particular fiber.

\section{CONCLUSION}

We have developed a new reproducible method, as far as different launch conditions are concerned, for measuring the MPD in a MMF and introduced a theoretical representation of it that can be used in designing short haul MMF communications systems. Using SI-MMF as a representative fiber, we showed the transition of the MPD along the fiber, revealing an unusual insertion loss-increasing phenomenon due to evanescent mode penetration into the cladding generation, and measured the EMD in the fiber. Because the proposed EAF calculation includes all 2-D FFP data, the resultant EAF curve reflects a nonaxisymmetric MPD, which is frequently observed in short reach optical communications. If device engineers or researchers measure their component's properties using a launched light distribution matching the predetermined EAF template, a fair evaluation can be performed. In future, moreover, if the input and output properties of all of the optical components in the system (e.g., MMFs, active and passive optical devices) are measured using a launched light distribution matching the predetermined EAF template, we can design and validate the system performance even if some components are replaced by ones from a different supplier or manufacturing process. Of course, the accuracy of the system design increases if multiple launched light distributions matching the predetermined EAF templates are used to measure the device properties. In addition, the transformation in EAF due to propagation and bending can be estimated by employing a theoretical power flow equation [39]. We call this concept, in which designers can design or evaluate the performance of communications systems using the optical power budget and the EAF curves, "Total MPD Management."

We would like to suggest using this method as a basic concept in the growing field of short-reach and high speed systems, which use MMFs and digital signal processing technologies, and are used particularly in the automotive industry, the home and industrial workplaces. Using "Total MPD Management" and limited launch conditions for the MPD, design engineers can perform high speed communications beyond the MMF's bandwidth distance limitation, which arises from the fiber's theoretical NA derived from the refractive indices of the core and the cladding. EAF measurement methods are currently being discussed for IEC standardization, and for standardization for related technologies worldwide.

\section{ACKNOWLEDGMENT}

The authors are grateful for technical discussions with Prof. R. Nagase, Chiba Institute Technology, Y. Hayashi, HAT lab, S. Kobayashi, TE Connectivity, T. Bolhaar, CommScope, R. Tao, UCL and Mr. S. Takahashi, POF Promotion.

\section{REFERENCES}

[1] C. A. Richard Pitwon, K. Wang, J. G.-Jones, I. Papakonstantinou, H. Baghsiahi, B. J. Offrein, R. D. Milward, and D. R. Selviah, "FirstLight: Pluggable optical interconnect technologies for polymeric electro-optical printed circuit boards in data centers," J. Lightw. Technol., vol. 30, no. 21, pp. 3316-3329, Nov. 2012.

[2] E. Ronald Freund, C.-A. Bunge, Nikolay N. Ledentsov, D. Molin, and C. Caspar, "High-speed transmission in multimode fibers," J. Lightw. Technol., vol. 28, no. 4, pp. 569-586, Feb. 2010.

[3] M. Kagami, T. Yamashita, M. Yonemura, and T. Matsui, "Light-induced self-written optical waveguides," IEICE Trans., vol. E90-C, no. 5, pp. 1061-1070, 2007.

[4] M. Stach, F. Rinaldi, M. Chandran, S. Lorch, and R. Michalzik, "Bidirectional optical interconnection at $\mathrm{gb} / \mathrm{s}$ data rates with monolithically integrated VCSEL-MSM transceiver chips," IEEE Photon. Technol. Lett., vol. 18 , no. 22 , pp. 2386-2388, Nov. 2006.

[5] R. Kruglov, J. Vinogradov, O. Ziemann, S. Loquai, J. Müller, U. Strauß, and C.-A. Bunge, "Eye-safe data transmission of $1.25 \mathrm{Gbit} / \mathrm{s}$ over $100-\mathrm{m}$ SI-POF using green laser diode," IEEE Photon. Technol. Lett., vol. 24, no. 3, pp. 167-169, Feb. 2012.

[6] S. J. Floris, B. P. de Hon, and T. Bolhaar, "Intrinsic attenuation in multimode fiber interconnects," in Proc. 61st Int. Wire Cable Symp. IWCS Conf., 2012, pp. 828-833.

[7] H. Yang, S. C. J. Lee, C. M. Okonkwo, S. T. Abraha, H. P. A. van den Boom, F. Breyer, S. Randel, A. M. J. Koonen, and E. Tangdiongga, "Record highspeed short-range transmission over $1 \mathrm{~mm}$ core diameter POF employing DMT modulation," Opt. Lett., vol. 35, no. 5, pp. 730-732, 2010.

[8] J. Carpenter, B. C. Thomsen, and T. D. Wilkinson, "Degenerate modegroup division multiplexing," J. Lightw. Technol., vol. 30, no. 24, pp. 3946-3952, Dec. 2012.

[9] L. Raddatz, I. H. White, D. G. Cunningham, and M. C. Nowell, "Influence of restricted mode excitation on bandwidth of multimode fiber links," IEEE Photon. Technol. Lett., vol. 10, no. 4, pp. 534-536, Apr. 1998.

[10] L. Raddatz, I. H. White, D. G. Cunningham, and M. C. Nowell, “An Experimental and theoretical study of the offset launch technique for the enhancement of the bandwidth of multimode fiber links," J. Lightw. Technol., vol. 16, no. 3, pp. 324-331, Mar. 1998.

[11] M. Webster, L. Raddatz, I. H. White et al., "A statistical analysis of conditioned launch for gigabit Ethernet links using multimode fiber," J. Lightw. Technol., vol. 17, no. 9, pp. 1532-1541, Sep. 1999.

[12] M. Hichem, D. Iyad, and A. Rabah, "Influence of wavelength and launching condition on frequency response of optical silica fibers," in Proc. IEEE 2nd ICTON Mediterranean Winter Conf. Marrakech, 2008, pp. 17-22.

[13] W. Daum, J. Krauser, P. E. Zamzow, and O. Ziemann, POF-Polymer Optical Fibers for Data Communication. Berlin, Germany: Springer-Verlag, 2001.

[14] Fibre Optic Communication System Design Guides-Part 11: Multimode Launch Conditions, IEC TR 61282-11, May 2012.

[15] P. Kolesar, "Standardization of measurement launch condition for multimode fiber cabling," in Proc. IEEE Avionics, Fiber-Opt. Photon. Technol. Conf., 2008, pp. 83-84.

[16] Measurement Methods and Test Procedures-Differential Mode Delay, IEC 60793-1-49, 2nd ed., Jun. 2006.

[17] P. Pepeljugoski, M. J. Hackert, J. S. Abbott, S. E. Swanson, S. E. Golowich, A. J. Ritger, P. Kolesar, Y. C. Chen, and P. Pleunis, "Development of system specification for laser-optimized $50 \mu \mathrm{m}$ multimode fiber for multigigabit short-wavelength LANs," J. Lightw. Technol., vol. 21, no. 5, pp. 1256-1275, May 2003.

[18] Fiber Optic Communication Subsystem Test Procedures-Part 1-4: General Communication Subsystems-Light Source Encircled Flux Measurement Method, IEC 61280-1-4, 2nd ed., Nov. 2009. 
[19] Optical Fibers-Part 1-40: Measurement Methods and Test ProceduresAttenuation, IEC 60793-1-40, 1st ed., Jul. 2001.

[20] S. Savović, A. Djordjevich, A. Simović, and B. Drljača, "Equilibrium mode distribution and steady-state distribution in 100-400 $\mu \mathrm{m}$ core step-index silica optical fibers," Appl. Opt., vol. 50, no. 21, pp. 4170-4173, 2011.

[21] S. Takahashi, T. Noda, and Y. Koike, "Restricted mode launch condition for graded index plastic optical fiber," in Proc. Int. POF Conf., 2008, pp. 124-126.

[22] R. Tao, T. Hayashi, M. Kagami, S. Kobayashi, M. Yasukawa, H. Yang, D. Robinson, H. Baghsiahi, F. A. Fernández, and D. R. Selviah, Equilibrium Modal Power Distribution Measurement of Step-Index Hard Plastic Cladding and Graded-Index Silica Multimode Fibers. San Francisco, CA, USA: SPIE, p. $93680 \mathrm{~N}, 2015$.

[23] S. J. Floris and B. P. de Hon, "Scalar versus vectorial impedance-angle formalisms for optical fibers," in Proc. IEEE Int. Conf. Electromagn. Adv. Appl., 2013, pp. 664-667.

[24] F. S. Tan, O. Sugihara, and T. Kaino, "Encircled flux-based optimized simple launch condition for standardization of multimode polymer optical waveguide evaluations," Opt. Exp., vol. 18, no. 23, pp. 23554-23561, 2010.

[25] S. K. Khijwania, F. D. Carter, J. T. Foley et al., "Effect of launching condition on modal power characteristics of multi-mode step-index optical fiber: A theoretical and experimental investigation," Fiber Integr. Opt., vol. 29 , no. 1 , pp. $62-75,2010$.

[26] M. Kagami, A. Kawasaki, and M. Yonemura, "Modal power distribution in short reach optical communications using step-index-type multimode optical fibers," in Proc. IEEE CPMT Sympo. Jpn., 2013, pp. 1-4.

[27] Examinations and Measurements-Encircled Angular Flux (EAF) Measurement Method Based on Two-Dimensional Far Field Data From Step Index Multimode Waveguide (Including Fibre), IEC 61300-3-53, 1st ed., Feb. 2015.

[28] D. Gloge, "Optical power flow in multimode fibers," Bell Syst. Tech. J., vol. 51, pp. 1767-1783, 1972.

[29] G. Jiang, R. F. Shi, and A. F. Garito, "Mode coupling and equilibrium mode distribution conditions in plastic optical fibers," IEEE Photon. Technol. Lett., vol. 9, no. 8, pp. 1128-1130, Aug. 1997.

[30] S. Savoić, B. Drljača, and A. Djordjevich, "Influence of launch-beam distribution on bandwidth in step-index plastic optical fibers," Appl. Opt., vol. 52, no. 6, pp. 1117-1121, 2013.

[31] D. Su, A. A. P. Boechat, and J. D. C. Jones, "Beam delivery by large-core fibers: Effect of launching conditions on near-field output profile," Appl. Opt., vol. 31, no. 27, pp. 5816-5821, 1992.

[32] T. Ishigure, M. Kano, and Y. Koike, "Which is a more serious factor to the bandwidth of GI POF: Differential mode attenuation or mode coupling?," J. Lightw. Technol., vol. 18, no. 7, pp. 959-965, Jul. 2000.

[33] R. Olshansky and S. M. Oaks, "Differential mode attenuation measurements in graded-index fibers," Appl. Opt., vol. 17, no. 11, pp. 1830-1835, 1978.

[34] M. Yonemura, A. Kawasaki, S. Kato, and M. Kagami, "Polymer waveguide module for visible wavelength division multiplexing plastic optical fiber communication," Opt. Lett., vol. 30, no. 17, pp. 2206-2208, 2005.

[35] M. Kagami, T. Yamashita, and H. Ito, "Light-induced self-written three-dimensional optical waveguide," Appl. Phys. Lett., vol. 79, no. 8, pp. 1079-1081, 2001

[36] Fibers Optic Connector Interface-Part 16: Type PN Connector Family, IEC 61754-16 ed1.0, Oct., 1999, or F07 Type connectors for optical fiber cables, JIS C5976, Jun. 1999.

[37] R. Olshansky and D. A. Nolan, "Mode-dependent attenuation of optical fibers: Excess loss," Appl. Opt., vol. 15, no. 4, pp. 1045-1047, 1976.

[38] Fiber Optics - Launch Condition Requirements for Measuring Multimode Attenuation, IEC 62614, 1st ed.Jul. 2010.

[39] S. Savovic' and A. Djordjevich, "Optical power flow in plastic-clad silica fibers," Appl. Opt., vol. 31, no. 16, pp. 7588-7591, 2002.

Manabu Kagami (M’04) received the B.E., M.E., and D.E. degrees in information engineering from Toyohashi University of Technology, Toyohashi, Japan, in 1982, 1984, and 1999, respectively.

In 1984, he joined Mitsubishi Rayon Corporation, where he worked on fiber components and crystal growth for light-emitting devices as a Research Engineer. In 1992, he joined Toyota Central R\&D Laboratories, Inc., Aichi, Japan, where he was a Research Manager of Applied Optics Laboratory from 2007 to 2012 and he is currently a Principle Researcher. From 2013, he has been appointed as a Visiting Professor of Toyota Technological Institute, Aichi, Japan, as well.
His research interests include the design of polymeric integrated optical circuits for interconnections and communication modules, optical network systems for automotive applications, and optical sensing device and systems. He has contributed to the development and standardization of test method for multimode optical fiber network devices at IEC/TC86/SC86B as an expert member

He received the Best Paper Award from IEICE in 2008. He is a Senior Member of the Optical Society of America and the Institute of Electronics, Information and Communication Engineers.

Akari Kawasaki, biography not available at the time of publication.

Masatoshi Yonemura received the B.E. and M.E. degrees in mechanical engineering from the Musashi Institute of Technology, Tokyo, Japan, in 1996 and 1998, respectively. In 1999, he joined Toyota Central R\&D Labs, Aichi, Japan, where he has been involved in research on plastic optical fiber communication. $\mathrm{He}$ is a Member of the Japan Society of Applied Physics.

Makoto Nakai received B.S. degree in electronics, information, and communication engineering and the M.S. degree in bioscience and biomedical engineering from Waseda University, Tokyo, Japan, in 2004 and 2006, respectively. He joined Toyota Central Research \& Development Laboratories, Inc., Aichi, Japan, in 2006. His current research interests include optical waveguide, fibers, and automotive Lidar systems.

Pablo V. Mena (S'94-M'99) received the B.S. (highest honors), M.S., and $\mathrm{Ph} . \mathrm{D}$. degrees in electrical engineering from the University of Illinois at UrbanaChampaign, Champaign, IL, USA, in 1994, 1995, and 1998, respectively.

He has held positions with RSoft Design Group, Ossining, NY, USA; Motorola, Schaumburg, IL, USA; Intel Corporation, Folsom, CA, USA; and Andrew Corporation, Orland Park, IL, USA. He is currently with Synopsys, Inc., Ossining, NY, USA, which acquired RSoft in 2012. His primary research interest has been the system- and circuit-level modeling of photonic devices, including quantum-well and vertical-cavity surface emitting lasers, standard and largecore multimode fiber, doped fiber amplifiers, and photodetectors. He has also investigated mixed-level simulation using system- and component-level modeling tools, design and simulation of photonic integrated circuits, simulation of next-generation optical systems based on multilevel and coherent modulation formats, and network-level modeling of end-to-end optical link performance. He has coauthored more than 30 journal and conference publications.

Dr. Mena is a Member of the IEEE, Tau Beta Pi, Eta Kappa Nu, and Phi Kappa Phi.

David R. Selviah (M'01) born in England, U.K., received the B.A. and M.A. degrees in physics and theoretical physics in 1980 and 1984, respectively, and the Ph.D. degree in photonic engineering in 2009 from Trinity College, Cambridge University, Cambridge, U.K.

He served internships at the Royal Aircraft Establishment, U.K., Texas Instruments, U.K. and CERN, Switzerland. From 1980 to 1983, he was with the Allen Clark Research Center, Plessey (Caswell), Ltd., U.K., (now Oclaro) From 1983 to 1986, he was with the Department of Engineering Science, Oxford University, U.K. He is currently a Reader in Optical Devices, Interconnects, Algorithms and Systems in the Optical Devices and Systems Laboratory of the Photonics Research Group in the Electronic and Electrical Engineering Department, University College London, London. He serves as a Consultant for UCL and VorteQ Consulting. He is the author of many published articles, keynote presentations, and patents. His research is generally carried out in collaboration with international companies and universities. His current research interests include multimode polymer optical waveguides, fibers and connectors for high bit rate communication in data centers, dispersion in single and multimode optical fibers, silicon photonic lasers, signal processing, image processing, pattern recognition, big data analysis, cloud computing, 3-D Lidar and photogrammetry, 3-D track classification, stimulation and monitoring neural behavior, data analysis for distributed acoustic sensors in oil and gas wells.

http://www.ee.ucl.ac.uk/staff/academic/dselviah

Dr Selviah is a Member of the Institute of Physics, Optical Society of America, European Optical Society and is a Chartered Physicist and Chartered Scientist. He represents the UK on the International Electrotechnical Commission Standards committees IEC TC86, SC86 WG4, SC86 WG6 and JWG9 covering optical fiber connectors, attenuation measurement techniques, and optical circuit boards. 